\title{
A Flexible Multiagent Parallel File System for Clusters
}

\author{
María S. Pérez ${ }^{1}$, Jesús Carretero ${ }^{2}$, Félix García ${ }^{2}$, José M. Peña ${ }^{1}$, and Víctor Robles ${ }^{1}$ \\ 1 DATSI. FI. Universidad Politécnica de Madrid. Spain \\ 2 Departamento de Informática. Universidad Carlos III de Madrid. Spain
}

\begin{abstract}
The emergence of applications with greater processing and speedup requirements, such as Grand Challenge Applications (GCA), involves new computing and I/O needs. Many of these applications require access to huge data repositories and other I/O sources, being the I/O phase a bottleneck in the computing systems, due to its poor performance. In this sense, parallel I/O is becoming one of the major topics in the area of high-performance systems. Existing data-intensive GCA have been used in several domains, such as high energy physics, climate modeling, biology or visualization. The I/O problem is not solved in this kind of applications. New approaches are required in this scene. This paper presents MAPFS, a multiagent architecture, whose goal is to allow applications to access data in a cluster of workstations in an efficient and flexible fashion, providing formalisms for modifying the topology of the storage system, specifying different data access patterns and selecting additional functionalities.
\end{abstract}

\section{Introduction}

Nowadays, there is a growing interest in the development of high-performance I/O systems, because the I/O phase has become a bottleneck in the computing systems due to its poor performance. In this sense, one of the major goals of high-performance computing is to provide an efficient access to data, being parallel I/O one of the most relevant issues in this field.

Currently, there are different parallel file systems, such as Galley [11], Parallel File System (PFS) [4] and Portable Parallel File System (PPFS) [8], which offer highperformance services to access resources. Many of these systems have been widely developed for parallel machines and are not suitable for clusters of workstations. Nevertheless, in parallel computing there is an increasing trend towards the usage of clusters, mainly because of their prices and their ease of integration. In this sense, the Parallel Virtual File System (PVFS) [3] is used in dedicated clusters of workstations.

On the other hand, parallel file system optimizations provide improved I/O operations. The usage of hints related to different aspects of data distribution and access patterns allows parallel file systems to increase the performance of these operations. Processes such as caching or prefetching are useful approaches used in addition with these last ones [12], [2]. The agent technology [5], [7] is a suitable framework for integrating these functions in the storage system, because of its adaptability to different domains and its capability to achieve process autonomy.

This paper presents MAPFS, a multiagent architecture, whose goal is to allow applications to access data in a cluster of workstations in an efficient and flexible fashion, 
providing formalisms for modifying the topology of the storage system, specifying different data access patterns and selecting additional functionalities.

The outline of this paper is as follows. Section 2 presents MAPFS as a flexible infrastructure for data-intensive applications in a cluster environment. Section 3 shows the results obtained for the evaluation of applications using MAPFS. Section 4 describes a comparison between MAPFS and PVFS. Finally, section 5 summarizes our conclusions and suggests further future work.

\section{MAPFS Overview}

The emergence of applications with greater I/O access requirements, named data-intensive applications or I/O-intensive applications, demands new I/O solutions. Examples of data-intensive and Grand Challenge applications [6] include data mining systems, data warehousing [9], high energy physics applications [15], and satellite data processing [1]. These applications may require access to data sources distributed among different nodes. Moreover, typical data-intensive applications require access to terabyte size datasets, which must be processed in an efficient way, in order to increase the performance of the applications executed on the cluster. Furthermore, data-intensive applications are very different depending on the kind of functional requirements and access patterns. It is critical for I/O system to be flexible enough to match these demands. The usage of hints, caching and prefetching policies or different data distribution configurations are optional features, which can reduce latency and increase I/O operations performance.

MAPFS [13] is a multiagent architecture, which provides flexibility in different aspects:

- System topology configuration: Ability to change system topology, setting the I/O nodes and their relationships. This feature is achieved through the usage of storage groups, which are described in section 2.2.

- Access pattern specification: Although MAPFS is a general purpose I/O system, it can be configured in order to adapt to different I/O access patterns [14].

- There are different reasons to allow some functionalities (such as caching or prefetching) to run in parallel on different nodes and even on the data servers. Moving executions to data servers may reduce network latency and traffic. The agent technology is useful in this scene, because of the agents properties, such as autonomy, proactivity and reactivity. MAPFS is composed of a multiagent subsystem, which is responsible of making several independent tasks.

\subsection{MAPFS Architecture}

MAPFS is based on a client-server architecture using general purpose servers. In the first prototype, NFS servers are used. NFS [10] has been ported to different operating systems and machine platforms and it is widely used by many servers worldwide. Data is distributed through the servers belonging to a storage group, using a stripe unit.

In the client-side, it is necessary to install a MAPFS client, which provides a parallel $\mathrm{I} / \mathrm{O}$ interface to the servers. 


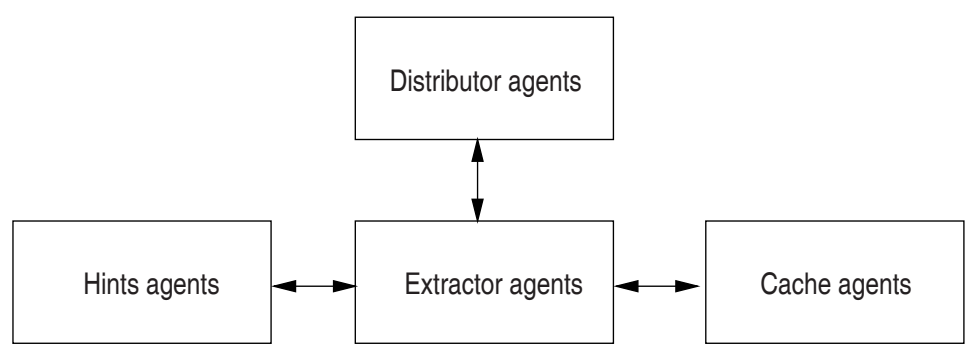

Fig. 1. Taxonomy of agents used in MAPFS

Additional multiagent subsystems provide several functionalities and they are executed on different nodes. Every storage group is associated to a multiagent subsystem. These multiagent subsystems use an agent hierarchy, which solves the information retrieval problem in a transparent and efficient way. The taxonomy of agents used in MAPFS is composed of:

- Extractor agents: They are responsible for information retrieval, invoking parallel I/O operations.

- Distributor agents: They distribute the work load to the extractor agents. These agents are placed at the higher level of the agents hierarchy.

- Caching and prefetching agents: They are associated with one or more extractor agents, caching or prefetching their data.

- Hints agents: They must study applications access patterns to build hints improving data access.

Figure 1 represents the relation among these agents. The taxonomy of agents can be extended to provide additional functionalities.

The most usual configuration is to run these subsystems on the data servers, helping to reduce network traffic. In this case, a major requirement is to install a technology that supports distributed execution of agents. MAPFS provides this optional functionality.

\subsection{Storage Groups}

The concept of grouping is fundamental in every aspect of the life. Edwin P. Hubble, which is considered the founder of the observational cosmology, said in the thirties that the best place for searching a galaxy is next to another one, describing the concept of galaxy grouping. Like in real life, computer science has a significant number of groupings, e.g. process group or user group.

A storage group is defined in MAPFS as a set of servers clustered as groups. These groups take the role of data repositories and can be built applying several policies, trying to optimize the accesses to all the storage groups. Some significant policies are:

- Grouping by server proximity: Storage groups are built based on the physical distribution of the data servers. Storage groups are composed of servers in close proximity to each other. This policy optimizes the queries addressed to a storage group because of the similar latency of messages sent to servers. 


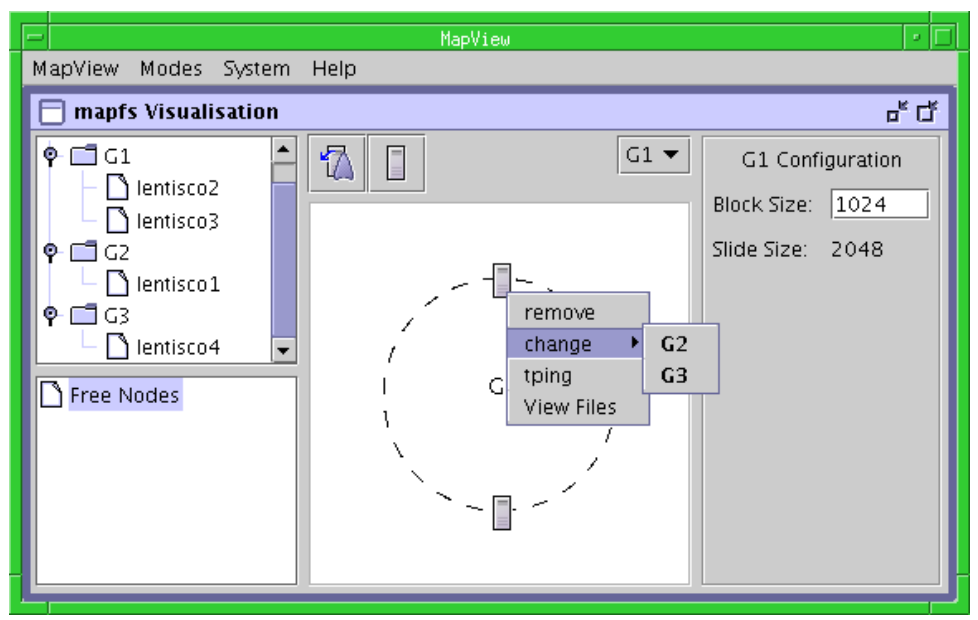

Fig. 2. MAPFS interface showing the system topology

- Grouping by server similarity: Storage groups are composed of servers with similar processing capacity. This policy classifies storage groups in different categories, depending on their computational and I/O power.

The main advantages of storage groups are:

1. Logic abstraction of the concept of storage server: As a partition is a logic abstraction of the physical disk, the storage group is also a logic abstraction of the storage server concept.

2. Dynamic management of servers: As we mentioned previously, the usage of storage groups provide dynamic management of servers through the MAPFS interface (see figure 2).

3. Efficiency of the storage operations: The policies used in the system provide a way of increasing the global efficiency of the system.

4. Load balancing: It is possible to use a concrete storage group in order to optimize system load balancing, depending on the load of the remaining storage groups.

5. Transparent migration: In addition to the MAPFS interface, the system can change the distribution of storage groups in a transparent way in order to optimize different aspects related to the system performance.

The system topology can be changed dynamically. In this case, data must be reconstructed, because the system must map the files stored in the servers of the previous topology to the new configuration. Data reconstruction degrades the performance of the I/O system. In order to avoid data reconstruction, MAPFS defines two types of storage groups, main storage groups and secondary groups, which form a lattice structure between them. Secondary groups are used for storing new data, avoiding the migration of the files data. In fact, this approach postpones data reconstruction until the system runs a de-fragmentation operation, which is used for deleting secondary groups and simplifying the storage system description. 


\subsection{Access Pattern Specification}

There are a huge number of applications using parallel file systems. These applications have very different requirements and data access patterns. Therefore, it is desirable that the underlying file system allows these applications to provide information about the layout of the data used by them. This information is given as hints, which are used for improving read and write operations performance. MAPFS uses these hints to access data. For example, storage systems using hints may provide greater performance because they use this information to decrease cache faults and to prefetch the data most probably used in next executions. In other words, the more information it has been used, the less uncertainty in the future access guesses and, therefore, the better prefetching and caching results.

In MAPFS, hints can be obtained in two ways:

1. Given by the user, that is, the user application provides to I/O system the necessary specifications.

2. Built by the multiagent subsystem. If this option is selected, the multiagent system must analyze the access pattern of the applications in order to build hints improving data access.

If hints are provided by the user application, it is necessary for the system to provide syntactic rules for setting the system parameters, which configure the I/O system. On the other hand, if the multiagent subsystem creates the hints, it is also necessary to store them in a predefined way. In any case, lexical and syntactic rules must be introduced in the system.

The system is configured through several operations, which are independent of the I/O operations, although these last ones use the former operations. The configuration operations are divided in:

- Hints Setting Operations: Operations for establishing the hints of the system. Therefore, they can set and get the values of the different fields of the hints.

- Control User Operations: Higher level operations that can be used directly by the user applications to manage system performance.

Figure 3 shows the three subsets of the Storage System API. As can be seen in the figure, there are three ways of accessing the Hints Setting Module:

1. The I/O operations may ask for hints values and even modify them.

2. The control user operations may modify the hints. This is the normal way for the user applications to interact with hints.

3. To make the system flexible, the Hints Setting Module may be accessed directly through the Hints Setting API. The multiagent system may build and modify hints through this interface.

The MAPFS I/O API was described in [13]. The hint setting operations are the following ones:

- Mapfs_Hints * mapHintsNew(int block_ident): This operation creates a new hint structure for the block with identifier block_ident. 


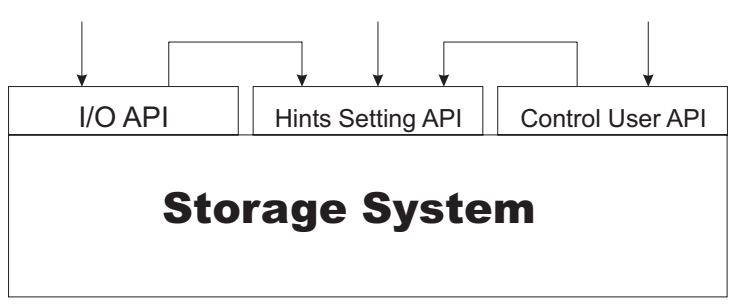

Fig. 3. Storage System API

- void mapHintsFree(Mapfs_Hints *hint): This operation releases a hint structure.

- int mapHintsSet(Mapfs_Hints *hint, int code_field, void * value): This operation modifies a field of the hint structure with a value. The operation returns 0 if successful and -1 otherwise.

- void * mapHintsGet(Mapfs_Hints *hint, int code_field): This operation returns the value of a concrete field of the hint structure. If the field is not defined, mapHintsGet() returns NULL.

Hints in MAPFS are built based on the concept of attribute. An attribute can take different values, which provides useful information for the I/O operations. The control user operations have a similar structure:

- Mapfs_CtrlUser * mapCtrlUserNew(Mapfs_Tuples *tupl): This operation creates a new Control User structure for a set of tuples represented by tupl.

- void mapCtrlUserFree(Mapfs_CtrlUser *ctrlUser): This operation releases a Control User structure.

- int mapCtrlUserSet(Mapfs_CtrlUser *ctrlUser, int code_field, void *expr): This operation modifies a field of the Control User structure with an expression. The operation returns 0 if successful and -1 otherwise.

- void *mapCtrlUserGet(Mapfs_CtrlUser *ctrlUser, int code_field): This operation returns the value of a concrete field of the Control User structure. If the field is not defined, mapCtrlUserGet() returns NULL.

Control User operations are higher level operation using expressions instead of simple attributes, which are translated to hints by the MAPFS system. These expressions are formed by boolean operations applied to attributes. An example is shown in the section 3 .

\section{MAPFS Evaluation}

In order to validate our implementation, it is necessary to evaluate its performance. Experiments were run on a cluster of four nodes Athlon $650 \mathrm{MHz}$, with $256 \mathrm{MB}$ of RAM memory, attached to a Gigabit network. This cluster constitutes our trial storage group. 


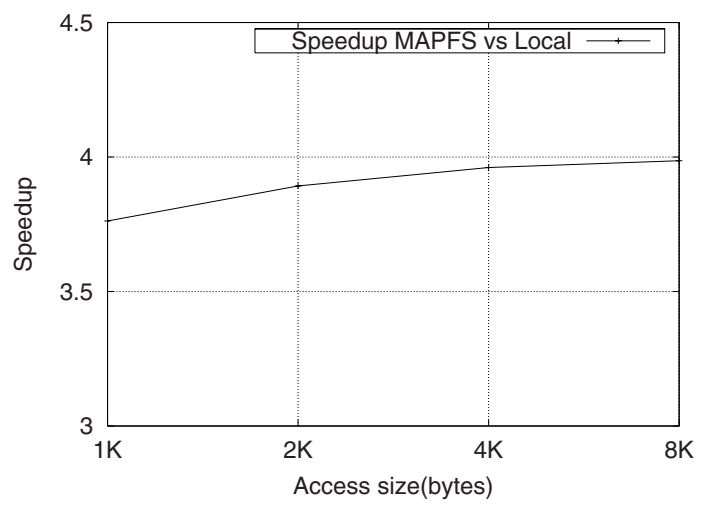

Fig. 4. Speedup of the MAPFS solution on a cluster of 4 nodes versus Local solution

Our experiment consists of four processes running a multiplication of two matrices, where the matrices are stored in the cluster, using MAPFS as underlying platform. The resultant matrix is also stored in a distributed fashion. A prefetching multiagent subsystem is used, which is responsible for prefetching rows and columns of the matrices. In this case, the hints provided by the applications are the indexes of the matrix row and the matrix column of the element calculated in every iteration. It is possible to prefetch data used in later executions, using this information. The control user structure provided by the application follows this expression:

$$
\text { row }=i \text { AND column }=j
$$

Therefore, the sequence of configuration operations made by the application are:

1. Mapfs_CtrlUser *mapCtrlUser = mapCtrlUserNew (data);

2. mapCtrlUserSet (mapCtrlUser, position, "row=i AND column=j") ;

This control user structure is translated to hints by the multiagent subsystem. Hints are used as data tags for processing the elements in an efficient manner, skipping nonrelated elements in the cache.

This experiment was compared to another one, which consists in multiplying the same matrices stored on the local disk through the usage of a traditional I/O system. The size of the matrices was $100 \mathrm{MB}$.

Figure 4 shows the speedup of the MAPFS solution versus local solution, varying the access size used in the I/O operations. As can be seen, the speedup is very close to 4 , the number of nodes, which is the maximum speedup, limited by the "Amdahl law". 


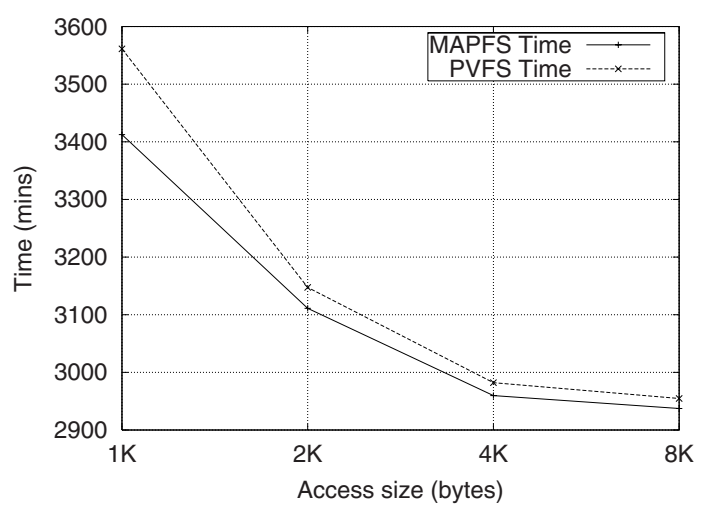

Fig. 5. Execution time of multiplication of two matrices with MAPFS and PVFS

\section{Comparison with PVFS}

The best way of evaluate MAPFS is to compare the performance of an application using this parallel file system and another parallel file system. PVFS [3] has been chosen due to the following reasons:

1. It is a parallel file system developed for Linux clusters.

2. It provides high bandwidth for concurrent read/write operations from multiple processes or threads to a parallel file.

3. It is robust and scalable.

4. It gives support for multiple APIs, including a native PVFS API, the UNIX/POSIX API and MPI-IO API. We have used the first one for implementing the application.

Again, we have compared a multiplication of two matrices distributed among different nodes both in MAPFS and PVFS. Figure 5 shows the execution time of the application using both parallel file systems. As can be seen in the figure, the difference is significant, although it decreases as the access size is increased. It is important to emphasize the fact that PVFS is a high-performance parallel file system.

\section{Conclusions and Future Work}

In this work we have presented MAPFS, a new multiagent architecture for high performance I/O in clusters. MAPFS provides the following properties: (i) system topology configuration; (ii) access pattern specifications by applications, and (iii) usage of a multiagent subsystem in order to run specific functionalities, such as caching or prefetching processes.

MAPFS has been compared to both a local solution and PVFS, a high performance parallel file system developed for Linux clusters, concluding that MAPFS provides a resultant speedup very close to the maximum one, improving the results of PVFS.

As future work, it would be interesting to evaluate the performance of the system with other applications, using more complex hints. 


\section{References}

1. Anurag Acharya, Mustafa Uysal, Robert Bennett, Assaf Mendelson, Michael D. Beynon, Jeff Hollingsworth, Joel Saltz, and Alan Sussman. Tuning the performance of I/O-intensive parallel applications. In Proceedings of the Fourth ACM Workshop on I/O in Parallel and Distributed Systems, May 1996.

2. Pei Cao, Edward W. Felten, and Kai Li. Implementation and performance of applicationcontrolled file caching. In Operating Systems Design and Implementation, pages 165-177, 1994.

3. P. H. Carns, W. B. Ligon III, R. B. Ross, and R. Thakur. PVFS: A parallel file system for linux clusters. In Proceedings of the 4th Annual Linux Showcase and Conference, pages 317-327, October 2000.

4. R. Esser and R. Knecht. Intel Paragon XP/S - architecture and software environment. In Proceedings of Supercomputer '93, Lecture Notes in Computer Science, Mannheim, Germany, 1993.

5. Stan Franklin and Art Graesser. Is it an agent, or just a program?: A taxonomy for autonomous agents. In Proceedings of the Third International Workshop on Agent Theories, Architectures and Languages, Springer-Verlag, 1996.

6. Grand Challenging Applications. http://www.mcs.anl.gov/projects/grand-challenges.

7. N. R. Jennings, K. Sycara, and M. Wooldridge. A roadmap of agent research and development. Autonomous Agents and Multi-Agent Systems Journal, 1(1):7-38, 1998.

8. James V. Huber Jr., Christopher L. Elford, Daniel A. Reed, Andrew A. Chien, and David S. Blumenthal. PPFS: A high performance portable parallel file system. In Proceedings of the 9th ACM International Conference on Supercomputing, pages 385-394, Jul 1995.

9. R. Kimball. The Data Warehouse Toolkit. John Wiley and Sons, Inc., 1996.

10. Network Working Group. NFS: Network File System Protocol Specification, March 1989. RFC 1094.

11. Nils Nieuwejaar and David Kotz. The Galley parallel file system. In Proceedings of the 10th ACM International Conference on Supercomputing, pages 374-381. ACM Press, May 1996.

12. R. Hugo Patterson, Garth A. Gibson, and M. Satyanarayanan. A status report on research in transparent informed prefetching. ACM Operating Systems Review, 27(2):21-34, 1993.

13. María S. Pérez, Félix García, and Jesús Carretero. A new multiagent based architecture for high performance I/O in clusters. In Proceedings of the 2nd International Workshop on Metacomputing Systems and Applications MSA’2001, September 2001.

14. María S. Pérez, Ramón A. Pons, Félix García, Jesús Carretero, and Víctor Robles. A proposal for I/O access profiles in parallel data mining algorithms. In 3rd ACIS International Conference on Software Engineering, Artificial Intelligence, Networking and Parallel/Distributed Computing, June 2002.

15. A. Shoshani, L. Bernardo, H. Nordberg, D. Rotem, and A. Sim. Storage management for high energy physics applications. Computing in High Energy Physics, 1998. 